\title{
Intelligent Management Functionality for Emergency Medical Applications Based on Cognitive Networking Principles
}

\author{
George Dimitrakopoulos, Marios Logothetis \\ Department of Informatics and Telematcis, Harokopion University of Athens, Athens, Greece \\ Email: gdimitra@hua.gr, marioslogo@gmail.com
}

Received November $14^{\text {th }}$, 2010; revised December 21 $1^{\text {st }}, 2010$; accepted December $28^{\text {th }}, 2010$.

\begin{abstract}
Telecommunications and information technology rapidly migrate towards the Future Internet (FI) era, which is characterized by powerful and complex network infrastructures, advanced applications, services and content, efficient power management as well as extensions in the business model. One of the main application areas that find prosper ground in the FI era, is medicine. In particular, latest advances in medical sciences are reflected on their capability to approach previously past-cure diseases, as well as to prevent the appearance and evolution of unpleasant situations. Those advances are often derived from interdisciplinary solutions to complex medical problems, supported by communications and electronics, which target fast, reliable and stable solutions to problems that are demanding in terms of velocity and accuracy. The goal of this paper is to present intelligent, knowledge-based management functionality capable of supporting emergency medical applications. An indicative emergency medical scenario is provided, along with extensive simulation results using the Network Simulator-2 (NS-2), for evaluating the performance of the proposed functionality.
\end{abstract}

Keywords: Future Internet (FI), Intelligent, Cognitive management, Electronic Medicine, Network Simulator-2 (NS-2)

\section{Introduction}

Over the last years, information and communication technology (ICT) have been lying at the forefront of international research and development efforts, aiming at the provision of innovative services and applications, tailored to user needs [1,2]. Latest trends refer to the migration of ICT towards the era of the "Future Internet" (FI) [3], which envisages 1) powerful network infrastructures, 2) the potential to provide multiple, advanced applications, services and content, by exploiting the powerful infrastructure, 3) "green" infrastructures in terms of efficient energy usage and 4) advances in the business model, socio-economics and security. The FI era promises easier overcoming of the structural limitations of telecommunication infrastructures and their management systems, through the adoption of cognitive networking principles [4-7], which further facilitate the design, development and integration of novel services and applications.

An area of applications where telecommunications and information technology find prosper ground in the FI era, is medicine. This is justified from the fact that the world of medical sciences has been undergoing an unparalleled evolution during the last years, this being reflected on the continuous development and enhancement of various solutions to medical problems. In this respect, as an outcome of worldwide research attempts, novel methods have been identified, which allow the diagnosis, treatment, even prevention of numerous, previously incurable, diseases. Moreover, the unstoppable development of ehealth products and applications can significantly improve the right of access to quality medicine, regardless of their personal condition and geographical location, allowing the selection of the appropriate health resource from anywhere at any time [8-11]. This is especially applicable in emergency situations, where timely retrieval of the necessary information might be of extreme importance.

Such advances have been facilitated through interdisciplinary research and development strategies. This refers mainly to the exploitation of recent findings in telecommunications and electronics, which cater for the development of complete systems that can really improve the patient's quality of life and reduce medical errors and 
costs. Indicatively, concepts such as the ubiquitous provision of applications at increasingly high bit rates, have paved the way for several innovative medical services and applications [10-11]. Additionally, the advent of the FI era [3] along with novelties in the network management domain through the exploitation of past interactions with the environment, in taking future decisions [12-13], is expected to facilitate several medical approaches, such as patient management technologies in telemedicine and remote diagnostics.

In this respect, the goal of this paper is to discuss on how wireless communication systems, enhanced with the advantages of cognitive networking principles, can serve as enablers for providing efficient and dependable, emergency medical applications, in the FI era. Several research attempts have been made in the past in the area of medical applications supported by wireless communications, without considering cognitive networking aspects. Some preliminary ideas on modeling quality of service (QoS) levels in e-health multimedia data are given in [14]. Bandwidth allocation for e-health applications and bandwidth aggregation is addressed in [15-16] to ensure their QoS guarantees. However, these schemes do not consider bandwidth aggregation over multiple wireless networks. Moreover, a discussion on how alternative technologies can support the QoS requirements of e-health applications is provided in [17-18], although no reference to integration of those alternative technologies is made.

The paper builds on the aforementioned proposals by presenting novel "Intelligent management functionality for emergency medical applications based on cognitive networking principles-i-MED”.

The contribution of this work is manifold:

First, the paper proposes intelligent management functionality that can aggregate and utilize knowledge and experience (thus exploit cognitive networking rules), so as to be able to quickly and efficiently respond to emergency contextual situations.

Second, the proposed functionality can provide satisfactory levels of abstraction during the provision of efficient and dependable medical services.

Third, the functionality contributes to the satisfaction of both, medical and patient requirements in terms of accuracy, reliability, unobtrusiveness and user acceptance, during critical situations.

The paper is structured as follows. First, the motivation for this work is presented, through 1) an outlook on the current wireless communications landscape, and 2) a description of an indicative emergency medical scenario that serves as a trigger for this work. Second, the paper describes the "i-MED" functionality, first at a high level view, and then in detail. Then, indicative simulation results using the Network Simulator 2 (NS-2) platform [19], are provided, for showcasing the performance of the proposed functionality. The paper concludes with summarizing remarks and some aspects for future reference.

\section{Motivation}

\subsection{The FI Era, Cognitive Management and Analogies with Medicine}

As mentioned above, the unstoppable research in telecommunications envisages their rapid migration towards the FI era. The FI era is associated with several requirements, which include the increased interest for wireless systems, the turning towards the provision of diversified applications, social networking, as well as the "prosumer" concept. Moreover, there is need for efficiency in resource provision (utilization, "green”, costs) and for resolving potential infrastructure problems: congestion situations, expand coverage when/where needed, efficiently offer localized applications and content [3].

From a technological perspective, in the FI era, wireless systems are regarded as systems having high-level structures with various system components, as illustrated in Figure 1. A Composite Wireless Network (CWN) represents a set of radio networks, which is operated by a Network Operator (NO) using a common network management system. Part of a CWN may be cognitive and can be managed by the NO, under a centralized cognitive management system. On the other hand, the Cognitive Control Network (CCN) enables the establishment of adhoc and mesh networks, catering for higher resource utilization, green decisions, lower costs, while it can also exploit autonomic capabilities.

In particular, regarding management, the objective of the FI management functionalities is to find the optimal network configuration of the managed segments, in order to serve the requests that they face. Essentially, they should produce service offers in response to the faced demand. In this respect, cognitive systems may be promising for facilitating the design, development and integration of novel services and applications in the FI era. In general, "a cognitive system is capable of retaining knowledge from past interactions with the external environment and decide upon its future behavior based 1) on this knowledge, 2) other goals and also 3 ) policies, so as optimize its performance" $[12,20]$. The operation of cognitive systems, called "cognitive cycle" is depicted on Figure 2(a).

An area of applications where cognitive systems could find prosper ground is electronic medicine (e-health). In this respect, Figure 2(b) shows the usual diagnostic-treatment cycle followed in the course of a medical incident handling. The whole cycle consists in an interacttion between the patient domain and the doctor domain. The doctor collects contextual information on the patient. This 

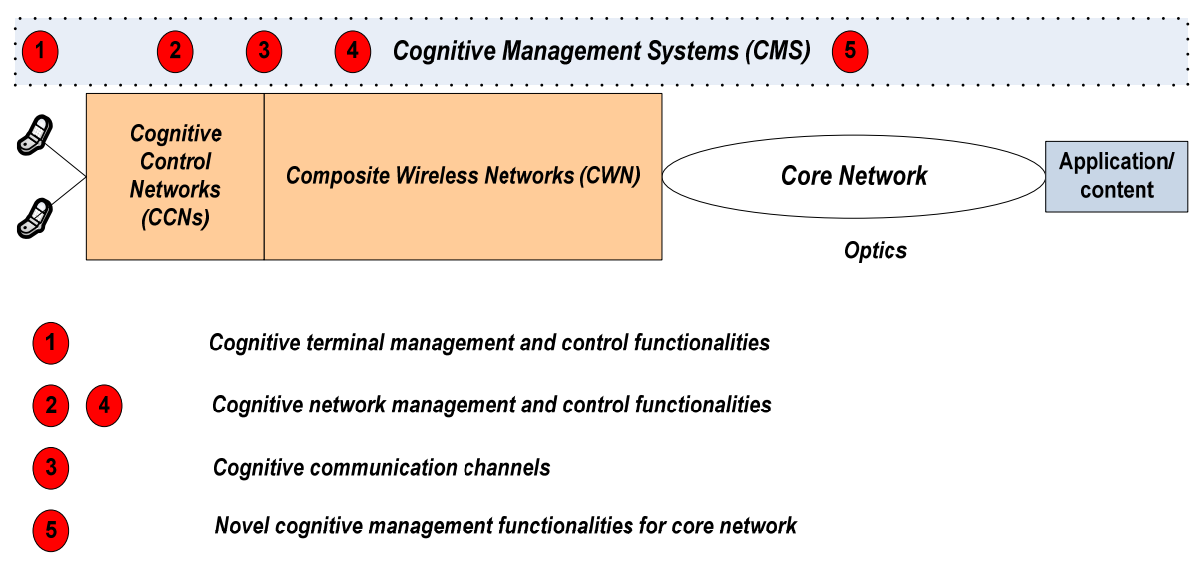

Figure 1. Realizing the FI world by means of cognitive systems.

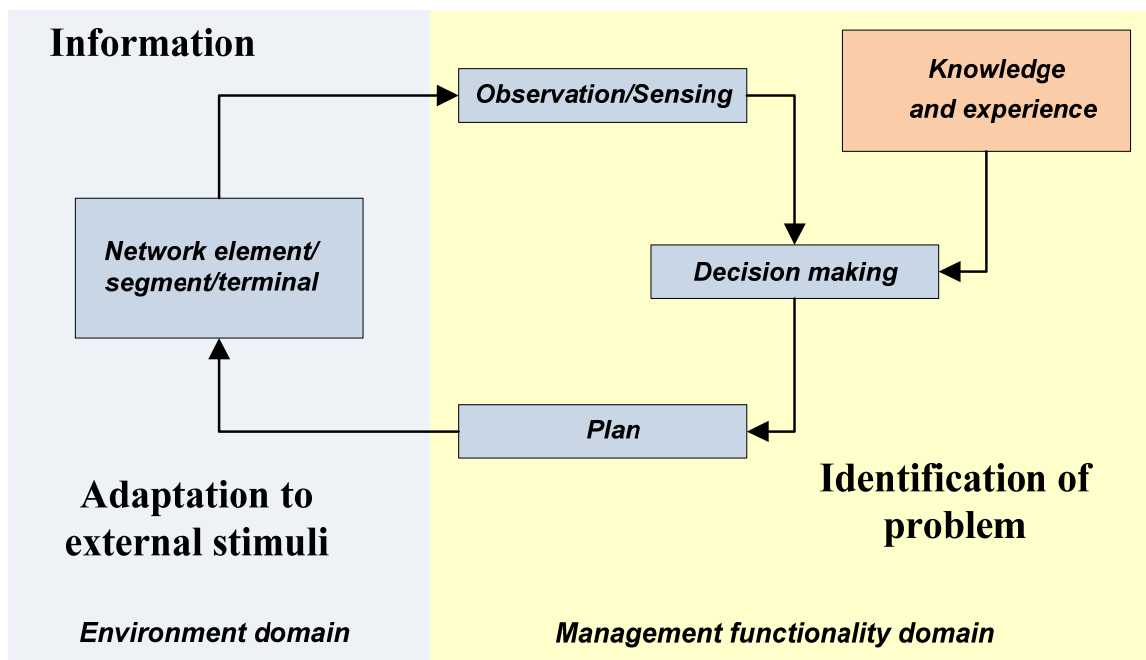

(a)

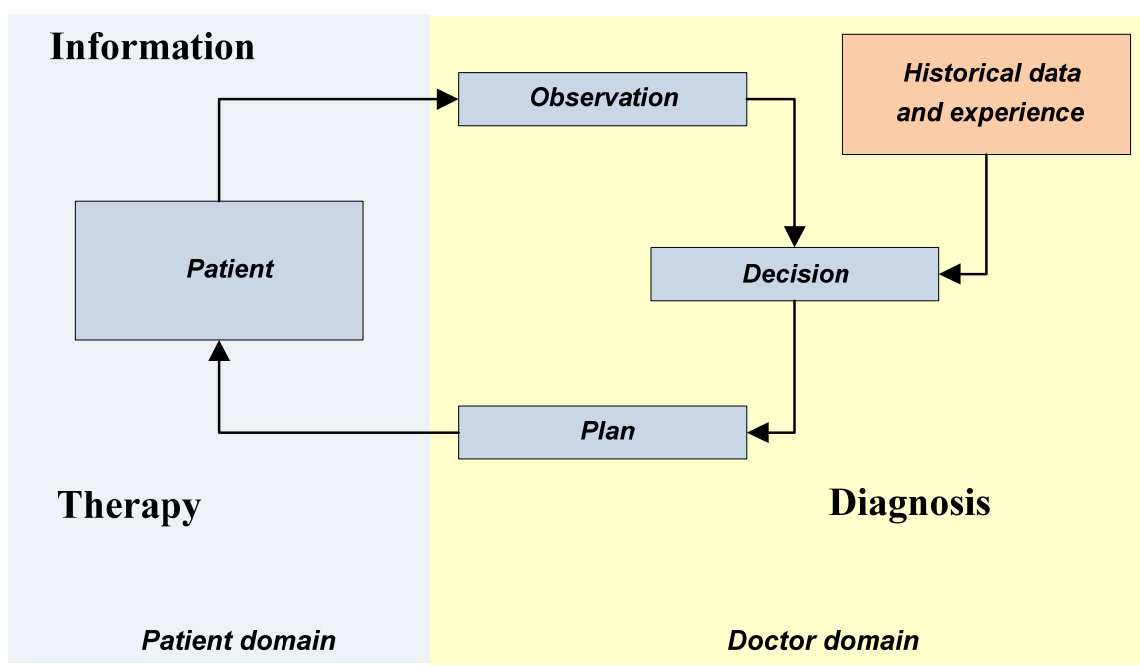

(b)

Figure 2. (a) The cognitive cycle in the case of wireless networks; (b) The diagnostic-treatment cycle: A cognitive-based approach. 
data, together with the patient's historical data, constitute the information to be analyzed by the doctor. The analysis results in the doctor's decision upon the most appropriate treatment manner to be applied to the patient in question. During the decision making process, the doctor considers specific goals and policies, as well as past knowledge and experience, which is derived from previous interactions with patients with similar incidents. So, the whole process can be reflected on a closed loop.

The diagnostic - treatment cycle finds several analogies with cognitive wireless networks and systems, as identified above, this being the basic motivation for this work. In particular, the doctor could be significantly facilitated by a system (management functionality) that keeps track of past actions, stores information on a "knowledge database" and provides this information as an input to the doctor, prior to decision making. At another level, cognitive management functionality may cater for fast and effective adaptations of the communication infrastructure to changing requirements, and thus guarantee unobtrusive communication during critical situations. This is exactly the focus of the paper.

In this respect, the next section presents an indicative scenario that shows how novel management functionality, enhanced with cognitive networking capabilities, may be needed for the provision of a medical application with faster transmissions, as well as higher reliability and availability.

\subsection{Indicative Emergency Medical Scenario}

This section aims at exemplifying the way in which cognitive management functionality can support a medical application, through an indicative emergency medical scenario, depicted on Figure 3.

The scenario begins with an ambulance having collected a patient from a place of an incident. No information is considered prior to this point (e.g. the call for an ambulance received by a telephone operator of the ambulance service). En-route towards the District General Hospital (DGH), pre-hospital emergency care is provided to the patient (e.g. basic/advanced life support), whereas also medical information is collected (e.g. bio signals, vital signs or medical DICOM images [21]) using the appropriate electronic instruments of the ambulance, and transmitted to the DGH through the utilization of ontologies, as defined by HL7 standard [22]. This information needs to be perfectly accurate, i.e. with the lowest possible level of packet loss, as well as of high quality, so as to be considered reliable for assessing the patient's condition.

As soon as the information reaches the DGH, it is used to potentially retrieve the patient's past medical data archived in a Medical Data Repository (MDR). The pa- tient's medical condition, as assessed and reported by the ambulance paramedics and the ambulance service staff, in conjunction with his/her medical data, is used for specifying the degree of urgency, as well as the potentially additional necessary medical procedures/measurement to be performed on the patient by the ambulance paramedics. Additionally, the MDR comprises also a Reference Context Repository (RCR), which states how similar incidents were tackled in the past. In this respect, the most appropriate doctor for the case is selected, according to the type of case, the case's degree of urgency and the DGH's availability, workload balance and suitability for the case. For example, in the case of a heart-related incident, a cardiologist needs to be immediately notified, whereas also the equipment of the relevant lab needs to be in order. This information is also communicated to the ambulance staff. Finally, on ambulance arrival at the emergency department of the DGH, the delivery of inhospital emergency medicine begins, while the case's data recorded by the ambulance service staff is made available to the emergency department's doctors. Emergency case's past medical data can also be made available to the emergency department's doctors on demand by accessing the health district's medical data repository at the DGH.

During such an emergency scenario, it is of utmost importance that information is transmitted at the most dependable manner. This is considerably difficult since the ambulance is constantly changing locations inside a versatile telecommunications environment. Consequently, intelligent management functionality is needed, for supporting the scenario. The enhancement of the functionality with knowledge and experience constitutes a fundamental step forward for increasing its efficiency and dependability.

\subsection{I-MED High-Level View}

In the light of the above, the cognitive management functionality for emergency medical applications (“i-MED”) aims at providing a seamless connectivity during such critical situations, while the ambulance is moving fast towards the DGH, as well as at building the basis for efficient emergency handling. To do so, it uses a set of inputs, utilizes optimization algorithms (that have been proposed and extensively analyzed in [23-26]) and provides an output consisting in the selection of the most ap- propriate (reliable) network operating parameters (wireless access systems and/or spectrum, power levels, bit rates for operation), so as to guarantee a seamless communication, which is of high significance. Furthermore, in order to be facilitated in making decisions, i-MED keeps track of past actions, so as to learn fromtheir implications, as discussed also in [27]. This is repeated in a machine learning 


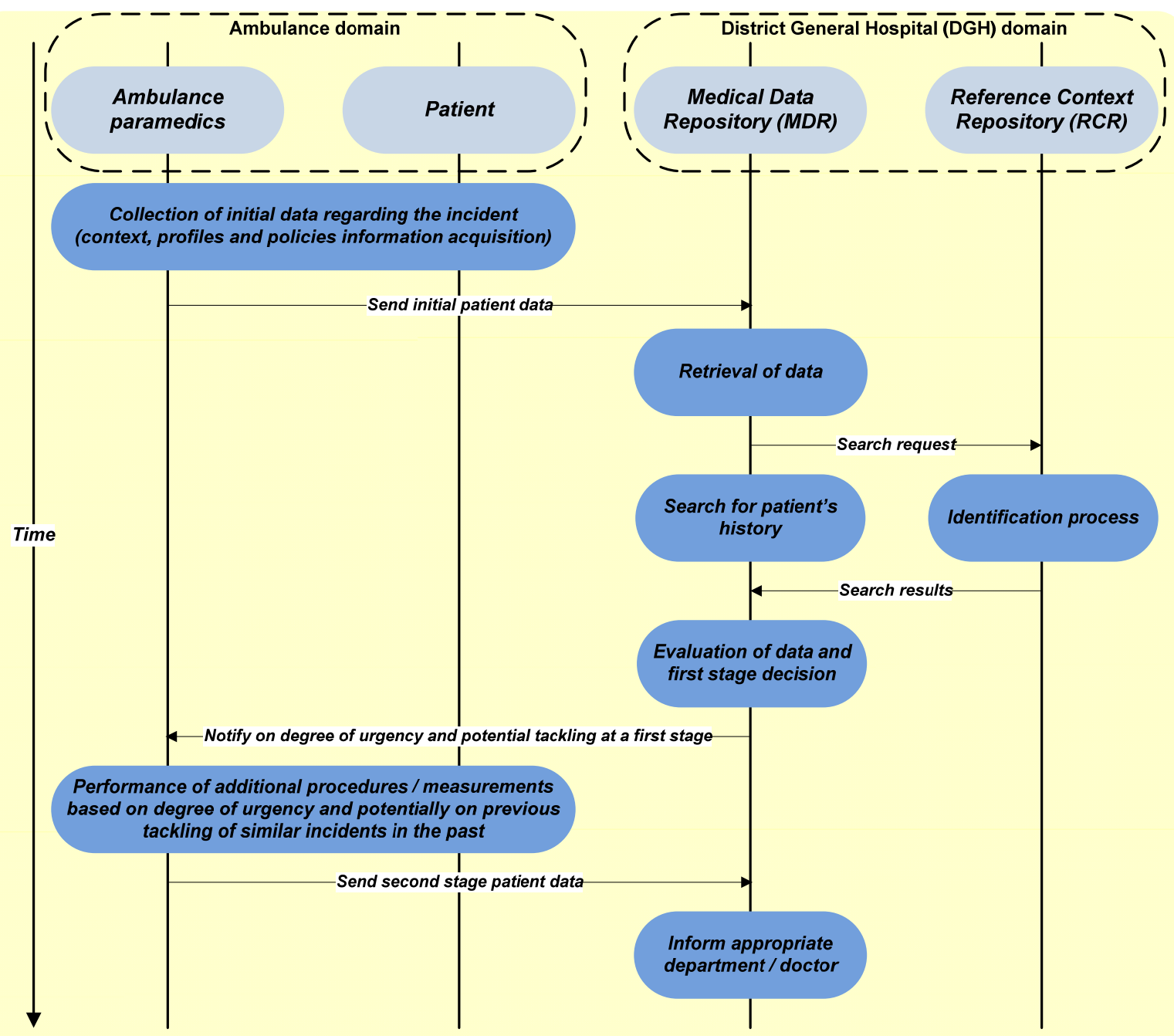

Figure 3. Emergency medical scenario.

process [20] that leads to cognition. In this respect, it reaches the appropriate decisions faster and even more efficiently, as will be shown herein. Last, through the cognitive process, i-MED is made even ready to proactively act in future relevant in- cidents, being able to predict the potential evolution of an incident, which might be of critical importance.

I-MED reflects a fully-distributed functionality, i.e., it is located on one hand inside the ambulance domain, aiming at the adaptation to the changing environment during the ambulance's heading towards the DGH, and on the other hand in the infrastructure domain, i.e., 1) in the DGH for the realization of the communication, and 2) in the Network Operator (NO) premises, enabling the NO to adapt the infrastructure on the basis of an emergency application. Moreover, it should be noted that the focus of i-MED is the utilization of cognitive management functionality, where the exploitation of knowledge helps the functionality reach faster, more stable and more reliable decisions. Moving one step further, cognition may also form part of the application itself, predicting the needs of the patient upon the arrival at the DGH. A high level view of the "i-MED” functionality is provided on Figure $\mathbf{4}$, while the next section presents it in detail.

\section{I-MED Detailed Description}

This section describes the "i-MED" functionality in detail, presenting its input, output and decision making method.

In general, as shown on Figure 4, the “i-MED” input includes information on the anticipated context, the infrastructure/ambulance profiles, as well as the policies imposed.

Context Acquisition. This functional block is responsible for monitoring the managed reconfigurable network elements/segments and provide any available information 


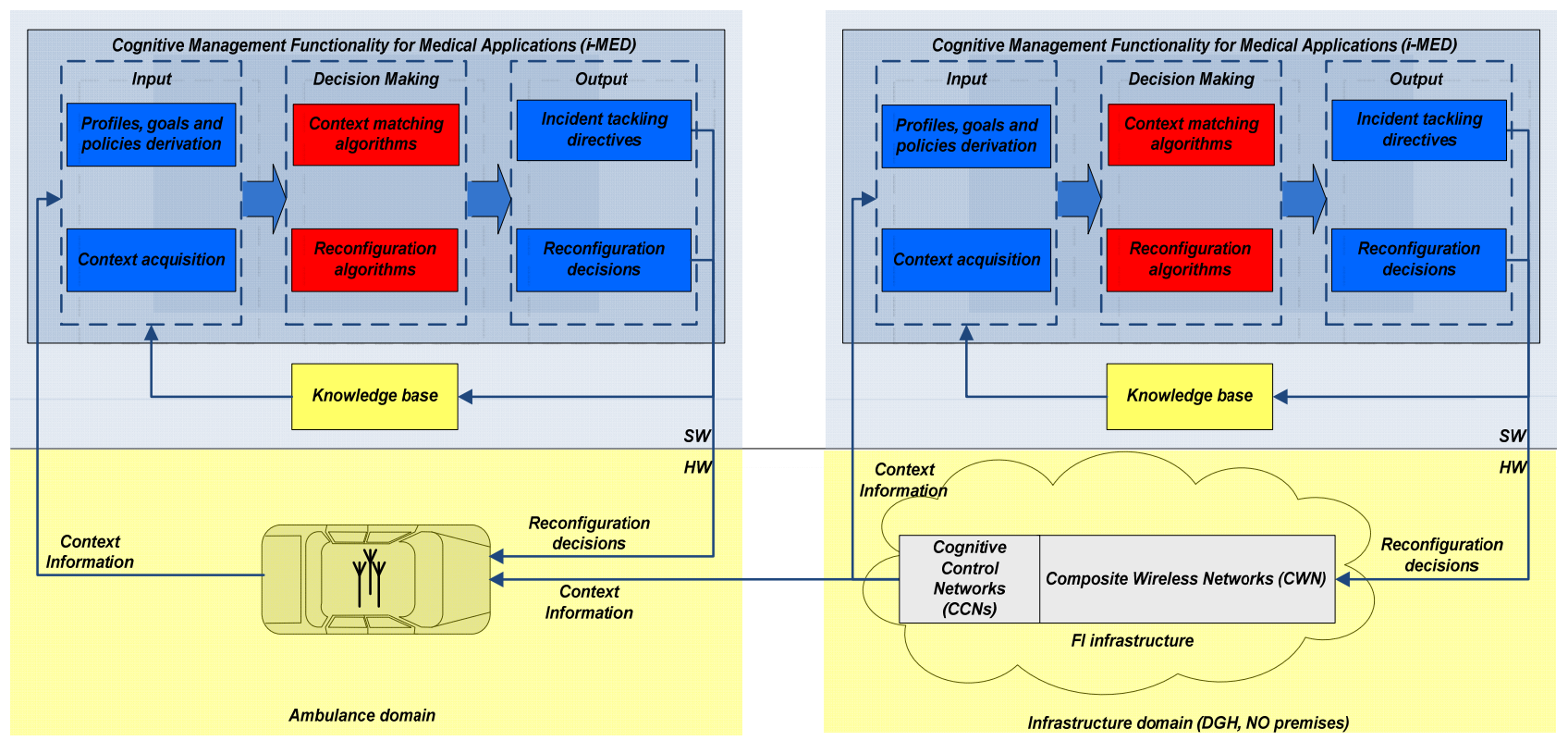

Figure 4. I-MED overview.

regarding the whole traffic in the network in terms of number of hosts, active sessions as well as information on the equipment that the sessions are initiated (reconfigurable wireless terminals), including the ambulance in question. Moreover, it is responsible to provide the service or application type (in general: entertainment, public, industrial administration, commercial, energy management, security, safety, educational, financial, etc.; in our case: medical) of the active sessions in the network as well as their priorities. In order to evaluate the current status of the network, several Key Performance Indicators (KPIs) need to be defined, mostly in terms of efficient network resources exploitation (delay, packet error rate, etc.), as will explained in the sequel, based on the sessions' service types and priorities. The main point for the operation of i-MED is the fact that medical applications appear to have a significantly high priority level compared to others (for accuracy, velocity and security reasons), as will be discussed in detail below.

Profile, goals and policies derivation. This functional block provides information on 1) the QoS levels that each user is willing to pay for each service type (the medical service appears on top of this list), 2) the security levels for each service type (the medical service is associated with higher security levels), 3) the ambulance's radio capabilities and 4) whether new software download is necessary in order the requested functionalities to be supported. Moreover, this block includes also information on the RAT profiles, which is necessary in order to include information regarding the capabilities (capacity, range, delay, supported service types, operational cost, security levels, power efficiency etc) of the available
RATs.

In addition, this functional block is essential for giving to a NO the potential to attribute high priorities to specific service types, one of which is an emergency medi$\mathrm{cal}$ service. It is quite easy to understand that service types like medicine oriented applications are high priority service types while the priorities of entertainment or commercial service types are the minimum especially when the traffic load is high. This can be efficiently exploited when taking decisions. Moreover, this functional block provides to a NO several options to configure 1) the maximum allowed QoS level per application depending on the traffic conditions, 2) billing functionalities based on the service types and the corresponding QoS levels (attributing the appropriate importance will make allocation more fair) and 3) the end-to-end domains involved.

Knowledge Base. All learning functionalities involved in i-MED are included in the knowledge base functional block. This block is responsible for 1) learning the most appropriate solutions regarding the functionalities or capabilities of the available services and/or applications, 2) learning the problems characteristics addressed in the past (e.g. past emergency situations) as well as their solutions provided after the optimization procedures, 3) being capable to identify whether a context or a problem has already captured or addressed in the past and provide the solution skipping optimization procedures and 4) keeping information regarding contexts addressed in the past [24]. Through using this information the system is 1) capable to predict future traffic conditions based on transition probabilities, 2) apply a priory the corresponding opti- 
mum configurations, as well as 3) predict the necessities of the patient upon the arrival at the DGH, based on tackling previous similar incidents.

Decision Making. This is the functional block where optimization takes place, considering the input from all the aforementioned blocks. Several algorithms can be employed regarding the following:

- Cross layer optimization. It includes reconfiguration actions for the majority of the protocol stack layers (data-link, network, transport and application). Based on the requested services the appropriate protocols are selected in order to increase the overall system performance while keeping the QoS levels the highest possible [23], which is extremely important in medical applications.

- Protocol parameters configuration. The optimum configuration of the protocol parameters are decided after the optimum protocol selection in order to guarantee the best possible network performance [25, 26].

- Software components optimum allocation. Several software components like services or applications should be efficiently allocated to the network entities (servers, proxies, routers etc.) of the system based on the traffic conditions and the demand for these software components [25,26].

- Service priorities optimization. Given that the priorities of the sessions' service types are available, they can be considered during the optimization process, so as to decide a) when the medical session will be served and b) what is the appropriate QoS level for the medical session.

- Context identification procedure. This procedure is responsible to identify whether a similar incident has already been addressed in the past and provide the corresponding solution skipping optimization procedures. In case that there is no identification, the optimization procedures will be triggered [24].

It should be noted that distributed context acquisition, and decision making at various degrees of distribution is enabled by the proposed architecture of the functionality, i.e., either in a collaborative manner under a NO surveillance, or autonomously on behalf of the ambulance. In general, the aforementioned components cooperate with each other, so as to generate knowledge from various sources and result in useful directives towards the ambulance and the infrastructure. The way this can be realized is the subject of the next section.

\section{Simulation Setup}

This section describes the way in which simulations have been prepared, in order to validate the performance of i-MED against conventional reconfiguration techniques.
First some general parameters of the simulation environment are presented. Second, some preliminary input data describing the emergency application considered (in accordance with the necessary i-MED input as described above), are provided. Finally, specific QoS metrics are presented in order to evaluate the proposed functionality.

\subsection{Preliminary Data}

The topology comprises a Flexible Base Station (FBS), i.e., a base station with 3 reconfigurable transceivers, each of which may operate with 2 wireless access systems in alternative, i.e., either in UMTS or in WiMAX mode. A total set of 20 terminals are served by this FBS. The range of UMTS is supposed to be $1000 \mathrm{~m}$, while the range of WiMAX is taken equal to $750 \mathrm{~m}$. The propagation model is set to Free Space, without effects from the environment, and the terrain profile is flat. Table $\mathbf{1}$ summarizes the general input parameters of the considered simulation environment. Last but not least, a set of applications are considered to be offered to the terminals through the FBS and further analyzed in the following section.

Moreover, results are obtained through simulations performed in NS-2 and run on a Pentium- $4.0 \mathrm{GHz}$ with 1.5 GB of RAM and a 32-bit operating system. NS-2 is a discrete event, standard simulation tool, which allows the accurate design and study of communication networks, devices, protocols and applications [19]. By providing the necessary input to NS-2, we can extract valuable results on the way in which i-MED can support an emergency medicine application. The simulation time was 60 minutes, whereas the results are depicted at the time scale of 1 min (exactly when the optimization and the adaptation occur).

3 scenarios are used for evaluating i-MED performance, mostly by showcasing the benefits of cognitive management functionality against conventional reconfiguration techniques during emergency situations. For the infrastructure-initiated scenario (scenario 1), the algorithms applied in the context of i-MED are the ones proposed in [23] (pure reconfiguration algorithm) and [24] (cognitive management algorithm). On the other hand, for the terminal (ambulance) - initiated scenario scenario 2), the algorithms proposed in [25] (cognitive terminal management) and [26] (reconfigurable terminal management) are recruited. Last, scenario 3 aims at exhibiting i-MED's per-

Table 1. General parameters of the simulation environment.

\begin{tabular}{lc}
\hline Area & $1250 \times 1250 \mathrm{~m}$ \\
\hline Simulation Time & $60 \mathrm{~min}$ \\
\hline Physical Characteristics & UMTS, Wimax \\
\hline \multirow{2}{*}{ Physical Layer Data Rate (Max) } & UMTS: 2 Mbps \\
\cline { 2 - 2 } & Wimax: $54 \mathrm{Mbps}$ \\
\hline Reception Power Threshold & $-95 \mathrm{dbm}$ \\
\hline
\end{tabular}


formance in terms of proactively identifying appropriate ways to tackle an incident using context matching algorithms [24].

\subsection{Application Aspects-Medical Sources Input}

Typical medical information is associated with the transmission of vital signs, such as electro-cardiogram (ECG), blood pressure and glucose, temperature and blood flow. Moreover, it includes live video, medical images, X-ray and voice communication between the ambulance and the DGH. A significant characteristic of medical data is the diversity in the period of transmission time. Hence it is possible to separate them in periodical and sporadic data. Periodic data may comprise vital signs such as ECG or heart rate. On the other hand, sporadic data are sent in an emergency situation when the patient's condition deteriorates, a case in which it may consist of high-band-width images and video.

Furthermore the services provided to users in the coverage area, include VoIP (provided to 15 users) and Internet Browsing (provided to 4 users). Internet browsing is modelled as a so called "Internet light Browsing". In particular, it uses HTTP protocol to download a web page of size equal to 500 bytes, plus an embedded, 5 small images of 250 bytes. Other important properties for the browsing application are listed in Table 2. The VoIP application is based on the G.711 [28] voice encoding scheme for both the caller and the callee. Further values for the attributes of the modelled VoIP application are given also in Table 2.

Moreover, the ambulance is assumed to be moving towards the DGH, according to the indicative scenario presented in Subsection 2.2. Two types of applications are considered for the ambulance depending on the demand in bandwidth. The services provided to the ambulance are image transfer and video conferencing for diagnosis purposes. Further details are given in Table 2.

\subsection{QoS Metrics}

As also stated in the introductory sections, the cognitive management proposed functionality is going to provide seamless connectivity during critical situations. Accordingly, in each of the scenarios we focus on specific QoS metrics, which are used to evaluate conditions and assist in coming up with useful results with respect to the proposed functionality. Particularly, in this simulation study, quality of service evaluation is carried out by the following performance metrics:

1) Delay (sec): which is the one way, end to end delay of data packets from the sending to the receiving node. It includes: a) processing delays e.g. voice packet compression/decompression, packetization etc. b) queuing and medium access delays in the AP as well as in the inter- mediate nodes, c) TRx delay of the AP and the interme diate nodes and d) the propagation delay for each conection between the AP and the destination node.

2) Data Dropped (Kbps): which is defined as the rate at which data is dropped due to full higher layer data buffers or because of too many retransmission attempts.

3) Throughput (Kbps): which corresponds to the total data traffic in bits per sec, successfully received by the destination excluding packets for other destination MACs, duplicate and incomplete frames.

4) Capacity (Mbps): which represents the total load in bits per sec in the simulated network.

All the above metrics are averaged to the set of terminals in the simulated network. Moreover, Table 3 summarizes the requirements for specific QoS metrics and for each of the considered applications. This table will be used as a reference throughout the rest of this paper and will assist in extracting conclusions from the derived statistics.

\section{Results and Discussion}

This section showcases the efficiency of the proposed functionality, through using 3 scenarios, as mentioned above.

Table 2. Traffic input parameters.

\begin{tabular}{|c|c|c|}
\hline \multirow{5}{*}{ Browsing } & \multicolumn{2}{|c|}{ Light, Protocol Version: Http 1.1} \\
\hline & \multicolumn{2}{|c|}{ Page Interval Time: Exponential 720 sec } \\
\hline & \multicolumn{2}{|c|}{ Pages Per Server: Exponential 10} \\
\hline & \multirow{2}{*}{$\begin{array}{l}\text { Page prope- } \\
\text { ties: }\end{array}$} & 1 constant part : 500 bytes \\
\hline & & 5 Small Images : 250 bytes each \\
\hline \multirow{5}{*}{ VoIP } & \multicolumn{2}{|c|}{ Encoder Scheme G.711 (silence) } \\
\hline & \multicolumn{2}{|c|}{ Compression/Decompression delay $0.02 \mathrm{sec}$} \\
\hline & \multicolumn{2}{|c|}{1 voice frame per packet } \\
\hline & \multicolumn{2}{|c|}{$\begin{array}{l}\text { Incoming and outcoming Conversation } \\
\text { environment: Land Phone - Quiet room }\end{array}$} \\
\hline & \multicolumn{2}{|c|}{$\begin{array}{l}\text { No control signalling (e.g. for setup/release) } \\
\text { included }\end{array}$} \\
\hline \multirow{2}{*}{$\begin{array}{c}\text { Video } \\
\text { Conferencing }\end{array}$} & \multicolumn{2}{|c|}{$\begin{array}{l}\text { High Resolution Video: } 704 \times 480 \text { pixels, } \\
24 \text { bits/pixel }\end{array}$} \\
\hline & \multicolumn{2}{|c|}{30 frames/sec } \\
\hline \multirow{2}{*}{$\begin{array}{c}\text { Image } \\
\text { Transfer }\end{array}$} & \multicolumn{2}{|c|}{ Inter-Request Time: 10 sec } \\
\hline & \multicolumn{2}{|c|}{ File Size: 500.000bytes } \\
\hline
\end{tabular}

Table 3. QoS requirements per application type.

\begin{tabular}{cccccc}
\hline \multirow{2}{*}{ Applications } & Technology & $\begin{array}{c}\text { Delay } \\
(\mathrm{ms})\end{array}$ & $\begin{array}{c}\text { Jitter } \\
(\mathrm{ms})\end{array}$ & $\begin{array}{c}\text { Bit Rate } \\
(\mathrm{Kbps})\end{array}$ & $\begin{array}{c}\text { Packet } \\
\text { Loss }\end{array}$ \\
\cline { 3 - 6 } & & Non real time \\
Image & $\begin{array}{c}\text { Nransfer } \\
\text { and Asymmetric }\end{array}$ & Medium & N/A & $<30$ & $0 \%$ \\
\hline $\begin{array}{c}\text { Video } \\
\text { Conferencing }\end{array}$ & $\begin{array}{c}\text { Real Time } \\
\text { and Symmetric }\end{array}$ & $<150$ & $<400$ & $<2$ Mbps & $<1 \%$ \\
\hline
\end{tabular}




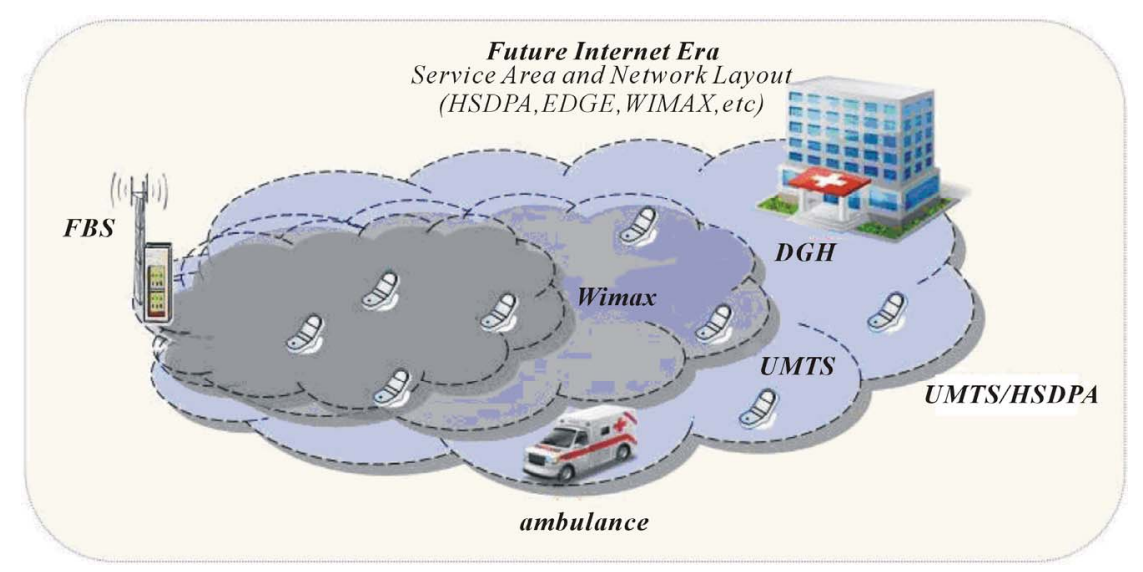

Figure 5. Service area.

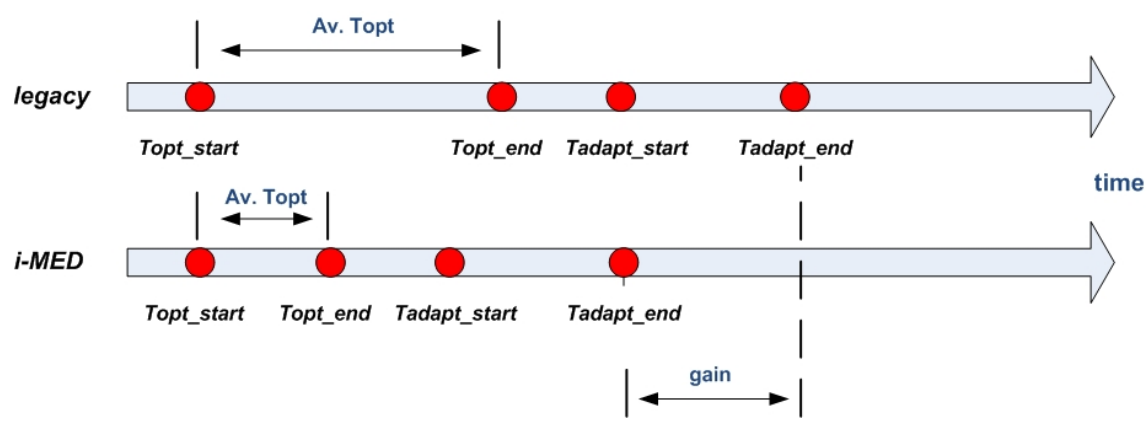

Figure 6. Optimization and adaptation time.

\subsection{Scenario 1: Initiation from the Infrastruture}

The scenario begins with the terminal (ambulance) that moves inside the service area of Figure 5, being provided a Video Conference service through UMTS. At some point, the "Context Acquisition" functional block of i-MED at the infrastructure domain (at the NO premises) identifies (through continuous monitoring of KPIs, such as delay, packet error rate, etc.) that there is need for guaranteeing a higher quality communication, due to an emergency medical situation. To do so, the "Decision Making" functional block of i-MED decides that a reconfigurable transceiver of the base station should switch to WiMAX, through the process described in [23], exploiting also the information from the "Profiles Management" and the "Policies Derivation" functional blocks. Moreover, if a similar incident has been confronted also in the past, i-MED will identify the appropriate solution through the "Knowledge Base" functional block faster, using algorithms such as the one proposed in [24].

In general, the whole process as depicted in Figure 6 is associated with an amount of optimization time (running time of the algorithm) and an additional amount of adaptation time (SDR adaptation time). We assume that the optimization time starts at the Topt_start point and finishes at the Topt_end point. Then, i-MED should com- mand the change of the operating wireless access system of the base station transceiver and inform the ambulance (and potentially other terminals, also), accordingly. This additional process starts at the Tadapt_start point and ends at the Tadapt_end point. As shown on Figure 7, the optimization time needed without the help of I-med, i.e., exploiting only the reconfiguration algorithm ([23]), is about $10.1 \mathrm{sec}$, which is comparatively larger (about $500 \%$ ) than the optimization time with the existence of i-MED (about $1.5 \mathrm{sec}$ ). This can be justified from the fact that i-MED exploits past knowledge and experience to reach the appropriate decision faster (with the use of cognitive algorithms such as the one described in [24]), which is of high importance in critical situations. This is achieved by completely avoiding running of the reconfiguration algorithms ([23]) and thus reducing the overall optimization time.

Moreover, Figure 8(a) and Figure 8(b) depict the average capacity (in Mbps) of the network segment (service area) during the simulation time for Video conference application. It is obvious that the average capacity is significantly increased with the choice of activating WiMAX in a transceiver. However, the i-MED gain lies in that the increased capacity is reached significantly faster (the curve in Figure $\mathbf{8 ( a )}$ is shifted towards the 


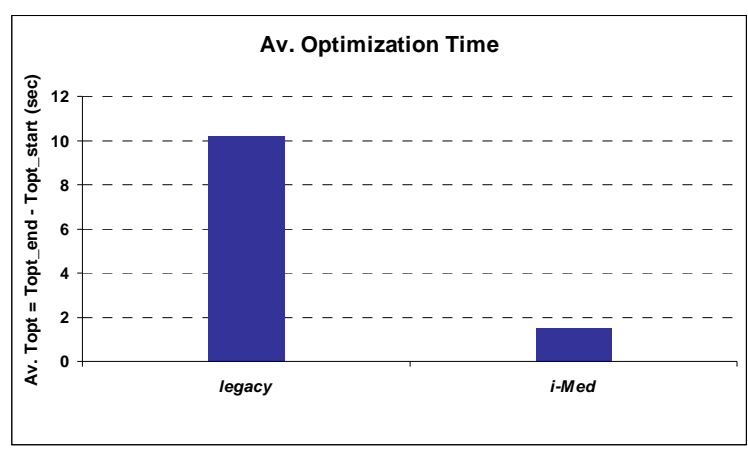

Figure 7. I-MED optimization time gain.

left), through the aforementioned decrease in the optimization time. Moreover, this contributes also to achieving a higher overall average capacity during the whole simulation, as shown on Figure 8(b). Furthermore, Figure 9(a) depicts the average capacity in case of image transfer through the network. As can be observed this application seems not to occur a prohibitive state for the network, due to the fact that the demand in bandwidth is low than the previous application. However as also depicted in Figure 9(b), image transfer application achieves a higher overall capacity in cell in case of i-MED functionality.

Finally, Figure 10(a) and Figure 10(b) depict the av-

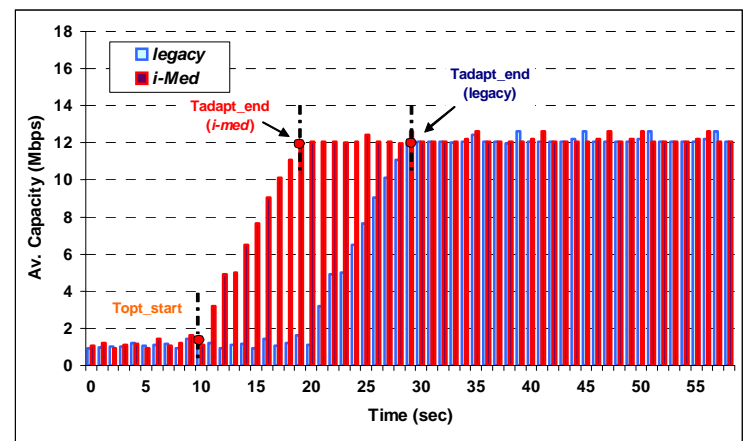

(a)

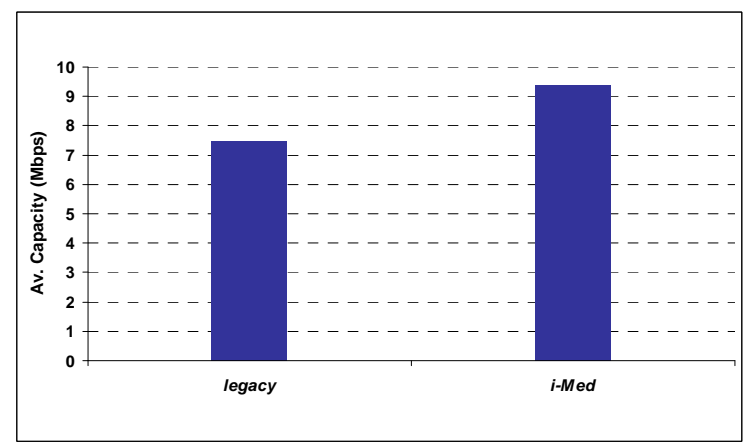

(b)

Figure 8. (a) Av. Capacity vs time; (b) Av. Capacity without (legacy) and with i-MED, for Video Conference Application.

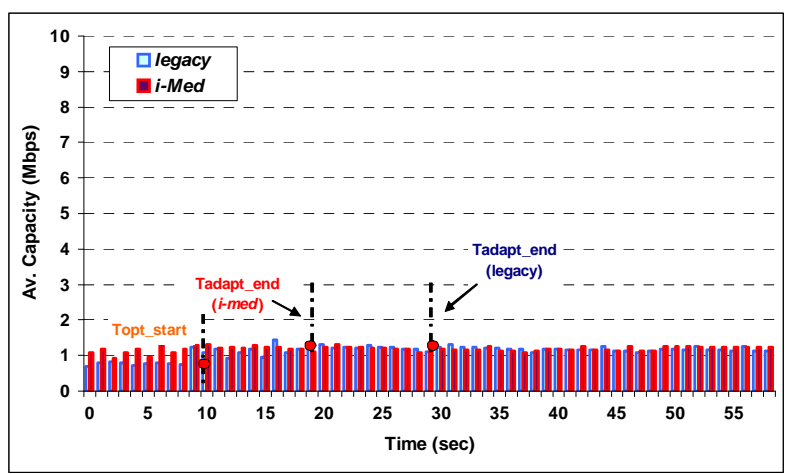

(a)

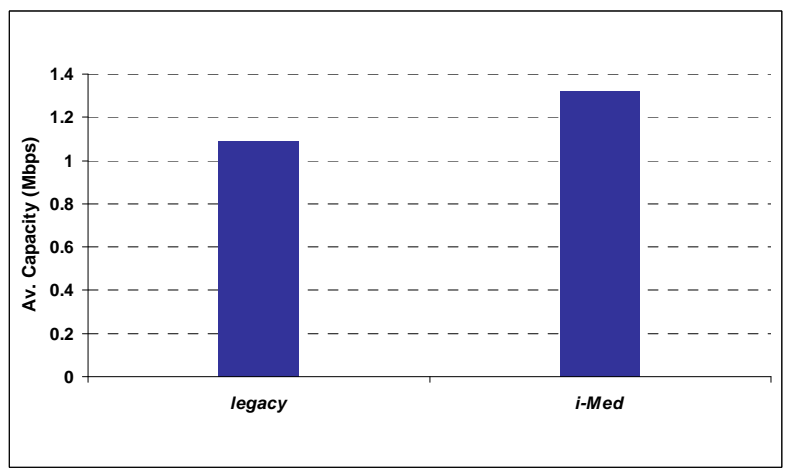

(b)

Figure 9. (a) Av. capacity vs time; (b) Av. capacity without (legacy) and with i-MED, for image transfer application.

erage data dropped (in Kbps) in the network segment during the simulation time for Video conference application. As can be observed in Figure 10(a), at the time when the optimization begins, the average data dropped increases, until the end of optimization. In this respect, since the optimization time is significantly decreased through i-MED (as explained above), the average data dropped becomes also decreased, accordingly. All in all, considering the whole simulation time, i-MED contributes to a total decrease of the average data dropped, as also shown on Figure 10(b). The same situation appears also in Figures 11(a),(b) for image transfer application, in which the average data dropped in the network for the whole simulated time is decreasing.

\subsection{Scenario 2: Initiation from the Ambulance}

This scenario considers the same as above input, being differentiated only by the fact that i-MED is now placed inside the ambulance (terminal). Again, the "Context Acquisition" functional block of i-MED (at the ambulance domain) identifies the necessity to better guarantee a seamless data transfer with the DGH (through the video conferencing service). Considering also the necessary information from the "Profiles Management" and the 


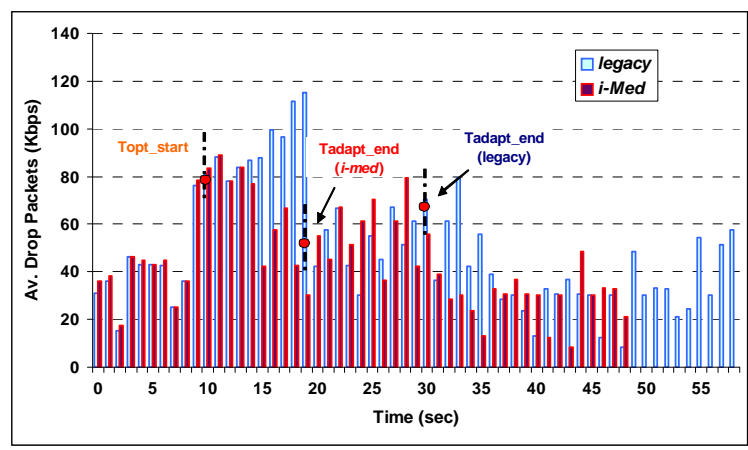

(a)

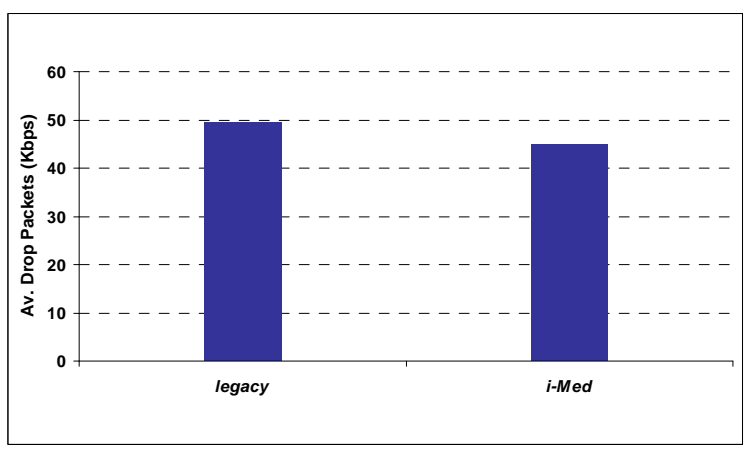

(b)

Figure 10. (a) Av. Data Dropped vs time; (b) Av. Data.

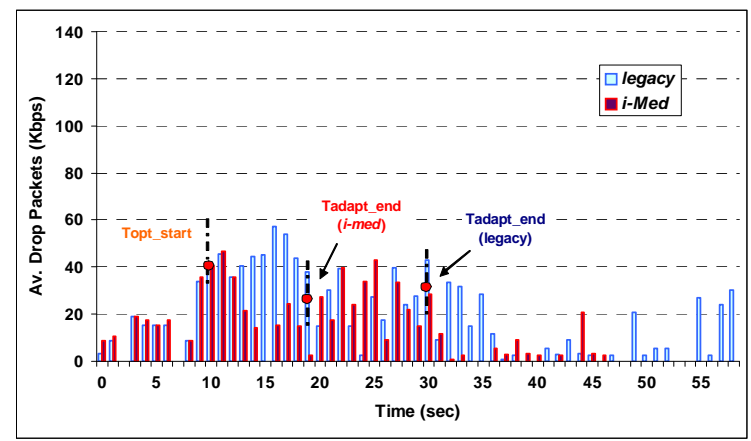

(a)

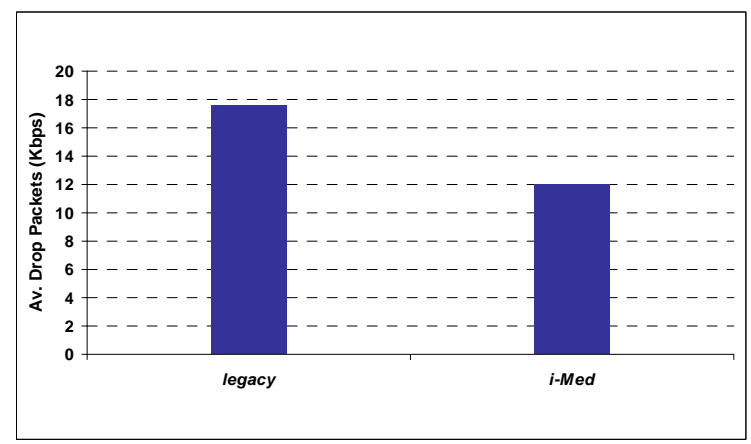

(b)

Figure 11. (a) Av. Data Dropped vs time; (b) Av. Data Dropped without (legacy) and with i-MED for Image transfer Application.

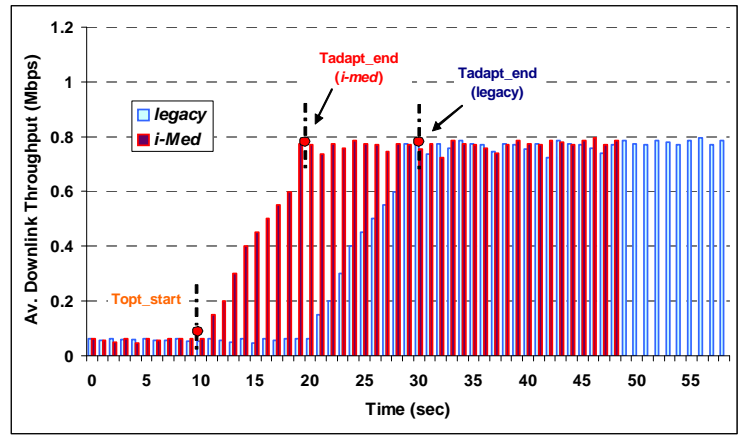

(a)

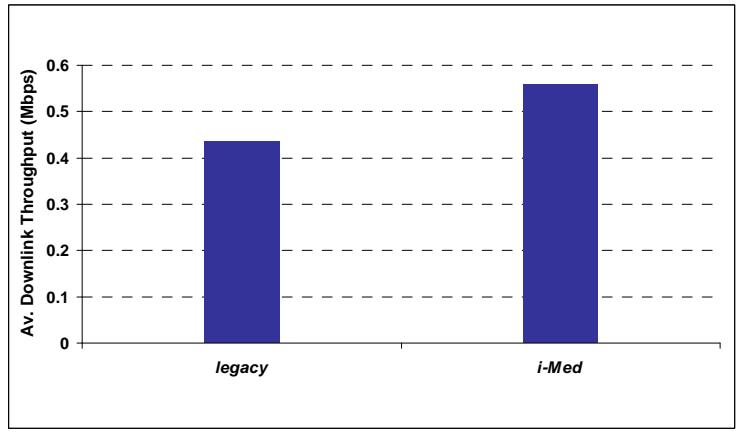

(b)

Figure 12. (a) Av. Downlink Throughput vs time; (b) Av. Throughput without (legacy) and with i-MED for Video conference application.

"Policies Derivation" functional blocks, the "Decision Making” functional block decides that the ambulance transceiver should switch from UMTS to WiMAX, employing reconfiguration algorithms, such as the one described in [26]. Moreover, if a similar incident has been confronted also in the past, i-MED will identify the most appropriate solution through the "Knowledge Base" functional block faster, using cognitive management algorithms, such as the one proposed in [25].

Figure 12 depicts the average downlink throughput of the ambulance (in Mbps), for Video conference, during the simulation time in the legacy (pure reconfiguration) case, as well as in the i-MED existence case. In general, the average downlink throughput depends on the wireless access system, packet size, as well as the time interval between packets. However, as can be clearly observed in Figure 12(a), the throughput increases in conjunction with the switching from UMTS to WiMAX. Moreover, the increased throughput is reached earlier in the case that iMED utilizes past information, since it avoids running the reconfiguration algorithms ([26]) and identifies the most appropriate solution (switching to WiMAX) faster. Therefore, I-med contributes also to achieving an increase in the overall average downlink throughput, compared to the legacy case, as shown on Figure 12(b). Furthermore Figures 13((a),(b)) depict the average downlink through put 


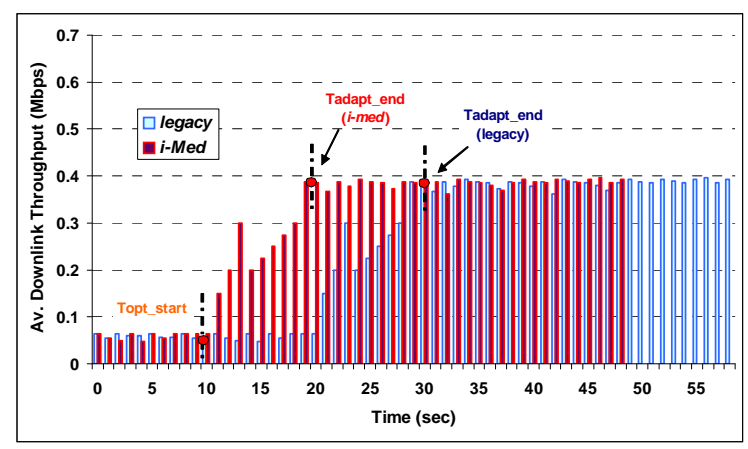

(a)

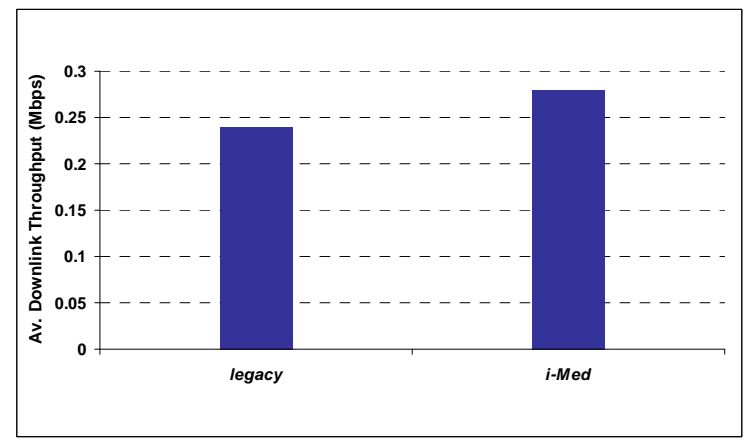

(b)

Figure 13. (a) Av. Downlink Throughput vs time; (b) Av. Downlink Throughput without (legacy) and with i-MED for Image transfer application.

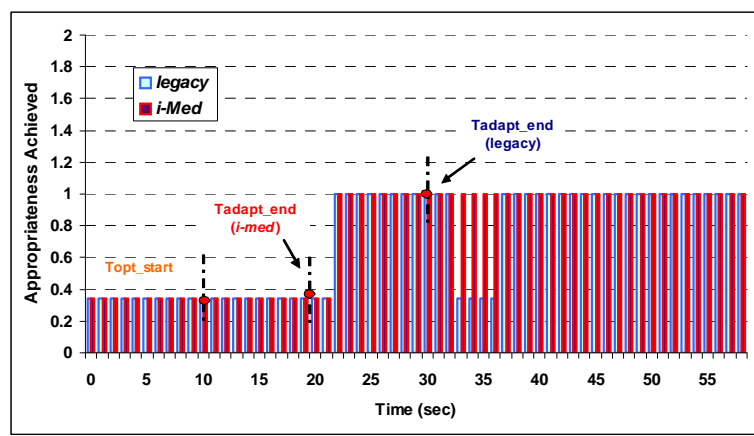

(a)

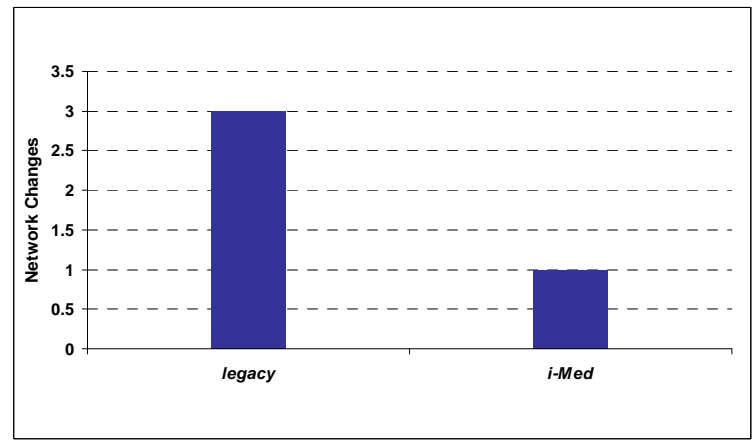

(b)

Figure 14. (a) Appropriateness Achieved vs time; (b) Network Changes without (legacy) and with i-MED. but this time for Image transfer application. As can be observed this application has the same tendency as the previous one and generally seems also to achieve with i-MED functionality an overall increase.

Last, Figure 14(a) depicts the degree of suitability (appropriateness) of the RAT, which the ambulance rates when it is inside the network [25,26]. Appropriateness comes also in line with the number of changes (hand overs) imposed without and with i-MED, shown on Figure 14(b). The only handover that is necessary occurs when i-MED indicate that the actual capabilities of the second network can guarantee a higher degree of reliability and dependability, which is of crucial importance when it comes to a demanding application, as in our case the medical one.

\subsection{Scenario 3: Prediction of Potential Incident's Necessities through Context Matching}

As described also in the indicative emergency medical scenario, i-MED is capable of identifying the best way to handle the medical incident, based on potential matching with similar incidents addressed in the past.

As the DGH gathers information, it initiates an identification process inside the CRP, which a Data Base (DB) is used, in order to remember the medical procedures that have been taken in every data set collected by the patient. Context matching can be based in well known techniques with the k-Nearest Neighbour(s) (k-NN) algorithm being a firm candidate. A pertinent solution which is based on k-NN and also exhibits non-prohibitive complexity has been provided in [24,29].

In order to validate our concept and give some evidence on the potentials arising from context matching functionality in our case of the emergency medical application, the overall end to end delay (in msec) is measured for a Data Base Access Application. The transaction inter-arrival time has been set equal to $30 \mathrm{sec}$ and the transaction size to 100 bytes. This delay, except from the metrics that have been presented in 4.3 , it also includes the response time from the DB query and entry, respectively.

Figure 15(a) depicts the average end-to-end delay of the application in the network segment during the simulation time. As can be observed, since the optimization time is significantly decreased through i-MED (as described above), the average data end-to-end delay is also decreased, accordingly. The same tendency appears also in Figure 15(b) for the overall end to end delay for the whole simulated time.

This proves in general i-MED's capability to 1) be ready to propose solutions to a medical problem based on previous tackling of similar incidents, as well as to 2) predict future similar situations. 


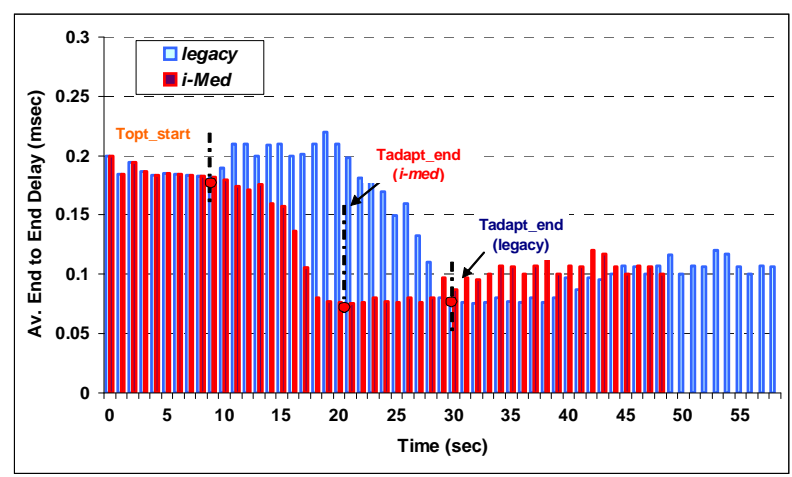

(a)

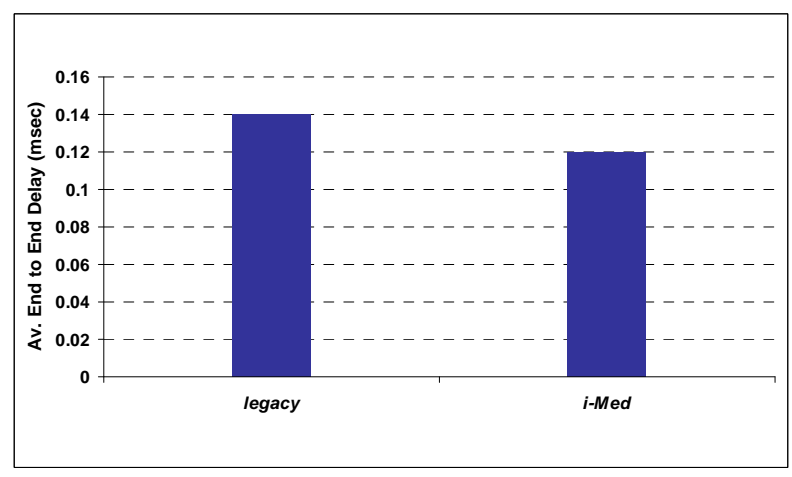

(b)

Figure15. (a) Av. End to End Delay vs time; (b) Av. End to End Delay without (legacy) and with i-MED.

\section{Conclusions}

The focus of this paper was the investigation of the potential contribution of cognitive wireless networks operating in the FI era, aiming at the enhancement of the efficiency and dependability of medical applications. In this respect, the paper has proposed novel intelligent management functionality (i-MED), based on cognitive networking principles, which is targeted at emergency medical applications. It has described the architecture of the proposed functionality and its operational principles, while it has also utilized two scenario variations for the extraction of simulation results that showcase i-MED's capability to provide fast and reliable solutions, utilizing the NS-2 simulator.

In general, i-MED can improve the performance of legacy reconfiguration management functionality in terms of throughput and capacity, since it can reach the appropriate decisions comparatively faster. In doing so, it can prove itself absolutely necessary in the case of critical situations, such as an emergency medical incident, where high gains in timely service provision and performance can be obtained. Therefore, i-MED enhances the performance of management functionality for medicine applications, by improving its overall levels of efficiency, re- liability and dependability.

Aspects of future work involve the further investigation in the exchange of information among i-MED functional blocks, in terms of interfaces definition and description. Moreover, novel algorithms will be explored in terms of accuracy and velocity, so as to further enhance the performance of i-MED. Last, cognition will be further extended to the application domain, so as to draw even more important medical conclusions (e.g. during the patient's transfer to the DGH) and propose alternative emergency treatment options, even with regards to surgery, based on previous knowledge and experience.

\section{REFERENCES}

[1] W. Hasselbring and R. Reussner, "Towards Trustworthy Software Systems,” IEEE Computer, Vol. 39, No. 4, April 2006, pp. 91-92.

[2] “Project End-to-End Efficiency (E3)," 7th Framework Programme (FP7) of the European Commission, Information and Communication Technologies (ICT), 2009. www.ict-e3.eu

[3] “European Future Internet Initiative (EFII)," 2010. http://initiative.future-internet.eu/

[4] S. Haykin, "Cognitive Radio: Brain-Empowered Wireless Communications," IEEE Journal on Selected Areas in Communications, Vol. 23, No. 2, February 2005, pp. 201220. doi:10.1109/JSAC.2004.839380

[5] R. Thomas, D. Friend, L. DaSilva and A. McKenzie, "Cognitive Drivers: Adaptation and Learning to Achieve End-to-End Performance Objectives,” IEEE Communications Magazine, Vol. 44, No. 12, pp. 51-57, December 2006. doi:10.1109/MCOM.2006.273099

[6] F. Jondral, "Cognitive Radio: A Communications Engineering View," IEEE Wireless Communications Magazine, Vol. 14, No. 4, August 2007, pp. 28-33. doi:10.110 9/MWC.2007.4300980

[7] J. Kephart and D. Chess, "The Vision of Autonomic Computing,” IEEE Computer, Vol. 36, No. 1, January 2003, pp. 41-50.

[8] Economic and Social Commission for Western Asia, "Promoting E-Health Applications towards an Information Society in ESCWA Member Countries," Western Asia Preparatory Conference for the World Summit on the Information Society (WSIS), Beirut, 4-6 February 2003.

[9] J. Grimson, W. Grimson and W. Hasselbring, "The SI Challenge in Health Care," Communications of the ACM, Vol. 43, No. 6, June 2000. doi:10.1145/336460.336474

[10] J.-C. Healy, "Future E-Health and Societal Needs," CISCO Public Services Summit, 2003.

[11] P.-A. Probst, "Role of ITU-T in E-Health Standardization Activities," WSC High Level Workshop on International Standards for Medical Technologies, Geneva, 26-27 February 2004.

[12] R. Thomas, D. Friend, L. DaSilva and A. McKenzie, 
"Cognitive Networks: Adaptation and Learning to Achieve End-to-End Performance Objectives,” IEEE Communications Magazine, Vol. 44, No. 12, December 2006, pp. 51-57. doi:10.1109/MCOM.2006.273099

[13] P. Demestichas, G. Dimitrakopoulos, J. Strassner and D. Bourse, "Introducing Reconfigurability and Cognitive Networks Concepts in the Wireless World: Research Achievements and Challenges," IEEE Vehicular Technology Magazine, Vol. 1, No. 2, June 2006, pp. 32-39. doi: 10.1109/MVT.2006.283572

[14] A. Bhargava, M. F. Khan and A. Ghafoor, "QoS Management in Multimedia Networking for Telemedicine Applications," Proceedings of 1st IEEE Workshop on Software Technologies for Future Embedded Systems (WSTFES’03), Hakodate, May 2003, pp. 39-42. doi:10.11 09/WSTFES.2003.1201357

[15] J. R. Gallego, A. Hernandez-Solana, M. Canales, J. Lafuente, A. Valdovinos and J. Fernandez-Navajas, "Performance Analysis of Multiplexed Medical Data Transmission for Mobile Emergency Care over the UMTS Channel," IEEE Transactions on Information Technology in Biomedicine, Vol. 9, No. 1, 2005, pp. 13-22. doi:10.11 09/TITB.2004.838362

[16] Y. Chu and A. Ganz, “A Mobile Teletrauma System Using 3G Networks,” IEEE Transactions on Information Technology in Biomedicine, Vol. 8, No. 4, 2004, pp. 456462. doi:10.1109/TITB.2004.837893

[17] P. Nanda and R. C. Fernandes, "Quality of Service in Telemedicine,” Proceedings of the 1st International Conference on the Digital Society (ICDS'07), Guadeloupe, January 2007, p. 2. doi:10.1109/ICDS.2007.35

[18] I. Reljin and B. Reljin, "Telecommunication Requirements in Telemedicine," Annals of the Academy of Studenica, Vol. 4, 2004, pp. 53-61.

[19] Network Simulator 2 (NS-2) Project. http://www.isi.edu/ nsnam/ns/

[20] T. Mitchel, "Machine Learning," McGraw-Hill, New York, 1997.

[21] P. Mildenberger, M. Eichelberg and E. Martin, "Introduction to DICOM standard,” European Radiology, Vol. 12,
No. 4, 2002, pp. 920-927.

[22] B. Orgun and J. Vu, "HL7 Ontology and Mobile Agents for Interoperability in Heterogeneous Medical Information Systems,” Computers in Biology and Medicine, Vol. 36, No. 7, July 2006, pp. 817-836.

[23] G. Dimitrakopoulos, K. Tsagkaris, K. Demestichas, E. Adamopoulou and P. Demestichas, "A Management Scheme for Distributed Cross-Layer Reconfigurations in the Context of Cognitive B3G Infrastructures," Computer Communications, Vol. 30, No. 18, December 2007, pp. 3807-3822.

[24] A. Saatsakis and P. Demestichas, "Context Matching for Realizing Cognitive Wireless Network Segments,” Wireless Personal Communications Journal, Vol. 55, No.3, September 2009, pp. 407-440. doi: 10.1007/s11277-0099807-z

[25] V. Stavroulaki, Y. Kritikou and P. Demestichas, “Acquiring and Learning User Information in the Context of Cognitive Device Management," Proceedings of IEEE International Conference on Communications 2009 (ICC 2009), Dresden, June 2009, pp. 1-5.

[26] P. Demestichas, A. Katidiotis, V. Stavroulaki and D. Petromanolakis, "Management System for Terminals in the Wireless B3G World," Wireless Personal Communications, Vol. 53, No. 1, 2010, pp. 81-109. doi: 10.1007/ s11277-009-9672-9

[27] G. Dimitrakopoulos, M. Logothetis and P. Demestichas, "Performance Evaluation of Cognitive Management Functionality for Emergency Healthcare Applications,” Proceedings of 6th Advanced International Conference on Telecommunications (AICT), Barcelona, May 2010, pp. 445-451.

[28] ITU-T Recommendation G.711, General Aspects of Digital Transmission Systems.

[29] A. Saatsakis, K. Tsagkaris, G. Dimitrakopoulos, A. Asvesta and P. Demestichas, "Emerging Management Strategies for the Introduction of Cognition in B3G Wireless Systems," Proceedings of ICT-Mobile Summit 2009, Santander, 10-12 June 2009. 